\title{
Microvascular Doppler ultrasound in children with acute pyelonephritis
}

\author{
Gayoung Choi ${ }^{1}$, Bo-Kyung Je${ }^{1}$, Doran Hong ${ }^{1}$, Jaehyung Cha ${ }^{2}$
}

${ }^{1}$ Department of Radiology, Korea University College of Medicine, Ansan Hospital, Gyeonggi, ${ }^{2}$ Medical Science Research Center, Korea University College of Medicine, Seoul, Republic of Korea

\begin{abstract}
Aims: To compare the diagnostic performance of microvascular Doppler ultrasonography (MVUS) to B-mode and conventional colour Doppler US (CDUS) for detecting acute pyelonephritis (APN) lesions in children. Material and methods: An IRB-approved retrospective study was performed. From July 2018 to January 2019, 41 APN lesions in 28 children (15 boys, 13 girls; age range, 1-196 months; mean age, 53 months) who underwent ${ }^{99 \mathrm{~m}} \mathrm{Tc}$-dimercaptosuccinic acid renal scintigraphy (DMSA) or contrast-enhanced computed tomography (CECT) and US including B-mode, CDUS, and MVUS were enrolled in this study. Three paediatric radiologists independently reviewed the B-mode, CDUS and MVUS images for the DMSA or CECT-proven APN lesions and evaluated the lesion visibility, lesion distinguishability and diagnostic confidence between the MVUS and CDUS images. Results: A total 41 of APN lesions were verified by DMSA (41 lesions) or CECT (3 lesions) during the same hospitalization period with renal US. Among 41 APN lesions, 52.8\% was visible on B-mode, $85.4 \%$ on CDUS, and $94.3 \%$ on MVUS ( $\mathrm{p}<0.001$ ). Comparing the extent and margins of the lesions, MVUS had better results than CDUS in $41.5 \%$ of the lesions, CDUS had better results in $6.5 \%$ and they were equal in $52 \%(\mathrm{p}<0.001)$. The diagnostic confidence of the APN lesions was higher for MVUS than CDUS in 36.6\%, higher for CDUS than MVUS in $4.9 \%$, and equal in the remaining 58.5\% $(\mathrm{p}<0.05)$. The interobserver agreement was fair to moderate. Conclusions: MVUS showed improved detectability of hypoperfused areas in paediatric APN and provided higher diagnostic confidence.

Keywords: pyelonephritis; paediatrics; ultrasonography; colour Doppler; microvascular imaging
\end{abstract}

\section{Introduction}

Acute pyelonephritis (APN) in children requires an immediate diagnosis for the appropriate treatment, in order to prevent complications, including irreversible scarring, renal hypertension and chronic renal failure. The diagnosis of APN in children, based on clinical and laboratory findings, is often challenging. Therefore, im-

Received 15.09.2020 Accepted 25.01.2021

Med Ultrason

2021, Vol. 23, No 2, 161-167

Corresponding author: Bo-Kyung Je, MD, PhD

Department of Radiology,

Korea University Hospital

123 Jeokgeumro, Danwongu, Ansan,

Gyeonggi 15355 Republic of Korea

Phone: 82-31-4125229

Fax: 82-31-4125224

E-mail: radje@korea.ac.kr aging studies are required [1,2]. For diagnosing APN in paediatric patients, ultrasonography (US), magnetic resonance imaging (MRI), renal cortical scintigraphy with ${ }^{99 \mathrm{~m}} \mathrm{Tc}$-dimercaptosuccinic acid (DMSA) scans and contrast-enhanced computed tomography (CECT) are available $[3,4]$.

Hypoperfusion is one of the major processes occurring during the development of APN, owing to cortical arteriolar vasoconstriction with inflammatory cells clogging the peritubular capillary and swelling of the renal interstitium after bacterial infection [5]. Depending on the superb detectability of the hypoperfused areas in the DMSA scans and CECT, these two modalities showed the highest sensitivity and specificity in diagnosing APN [1]. However, they have disadvantages for use in paediatric patients, such as radiation exposure and invasiveness that requires an intravenous injection. MRI is also sensitive and specific. However, it involves paediatric 
patient sedation, long examination times and high costs. In comparison, US has many advantages as the primary diagnostic modality for paediatric APN, such as an easy application, good cost-effectiveness and safety not requiring radiation exposure [6]. In spite of these advantages of US, the diagnostic sensitivity of US has been reported as 20 to $69 \%$, much lower compared with the other techniques $[1,7,8]$.

However, US technology has evolved and new emerging techniques have improved the diagnostic performance of US. The sensitivity and specificity of conventional colour Doppler US (CDUS) for detecting hypoperfused areas have also been improved. Recently, a new innovative Doppler technique was introduced; the technique can depict tissue microvessels with slow blood flow that are removed along with clutter artifacts by conventional colour Doppler filters $[9,10]$. This microvascular Doppler US (MVUS) is a promising method to demonstrate tissue microvasculature and distinguish vascular lesions $[10,11]$.

To our knowledge, there has been no published study on the diagnostic performance of MVUS in detecting APN lesions compared to CDUS. In this study, we retrospectively reviewed the US features of 41 APN lesions that were confirmed by DMSA scans or CECT. The purposes of this study were to evaluate the diagnostic capabilities of B-mode, CDUS and MVUS images for paediatric APN and compare the diagnostic performance of MVUS and CDUS techniques.

\section{Materials and methods}

\section{Subjects}

The Institutional Review Board approved this retrospective study and waived the requirement for informed consent. From July 2018 to January 2019, 424 children underwent renal US at our institution. With the exclusion of renal US requested for non-infectious reasons and follow-up renal US for previous urinary tract infections (UTI), a total of 123 renal US examinations were performed for the suspicion of a first febrile UTI episode. Among them, the patients who underwent DMSA scans or CECT during the same hospitalization period with renal US were selected. Then, the patients with renal perfusion defects on DMSA scans or CECT were included. Finally, 41 APN lesions in 28 children (15 boys, 13 girls; age range, 1-196 months; mean age, 53 months) were enrolled (fig 1).

\section{Imaging studies}

A paediatric radiologist with 15 years of experience performed the renal US examinations with a commercially available US scanner (RS 85, Samsung Medison, Seoul, Korea), equipped with linear (4-15 MHz) and

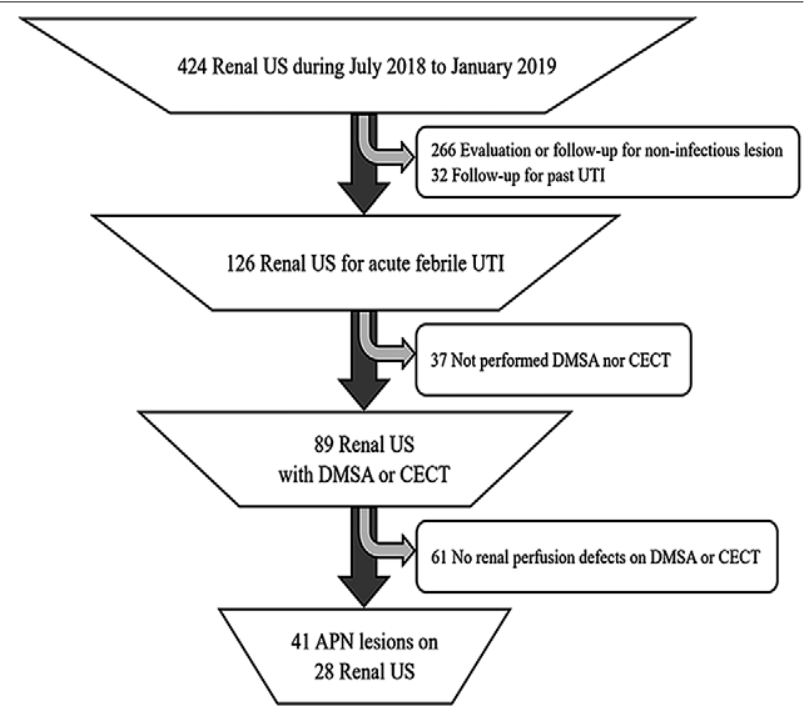

Fig 1. Enrolment of subjects. UTI: urinary tract infection; DMSA scan: ${ }^{99 \mathrm{~m}} \mathrm{Tc}-$ dimercaptosuccinic acid scan; CECT: contrast-enhanced CT including the kidney

convex transducers (1-7 MHz). The MVUS examination (called MV-Flow ${ }^{\mathrm{TM}}$ ) was performed with these transducers. All renal US examinations included B-mode, CDUS and MVUS images in both the longitudinal and transverse axes of each kidney, obtained in the same area. The three scanning modes were readily switchable by pressing buttons on the scanner control panel. The depth and focal zones were identical and other scanning parameters were applied as provided. No sedatives or other invasive interventions were given, but some children with irritability were helped by extra-assistance including video watching during the US examinations.

A DMSA scan was performed 2 hours after intravenous injection of ${ }^{99 \mathrm{~m}} \mathrm{Tc}-$ labeled DMSA in the routine protocol (1-2 $\mathrm{mCi}$ according to body weight) using a tomographic gamma camera (Infinia, GE Healthcare, Milwaukee, WI, USA), which included anterior, posterior, and both posterior oblique images. All patients were asleep with oral chloral hydrate $\left(\right.$ Pocral $^{\mathrm{R}}$ syrup; Hanlim, Korea) during the examination.

A CECT scan was obtained 70 seconds after the injection of intravenous iodine contrast media with 128-slice spectral CT (IQon Spectral CT, Philips Healthcare, Cleveland, OH, USA). The typical $\mathrm{kVp}$ of CECT was determined according to the body weight of each patient: $80 \mathrm{kVp}$ for children under $10 \mathrm{~kg}, 100 \mathrm{kVp}$ for $10-30 \mathrm{~kg}$, $120 \mathrm{kvP}$ for over $30 \mathrm{~kg}$. Three patients underwent CECT and none required sedation.

\section{Evaluation of images}

DMSA scans and CECT were used as the verifying modalities for the APN lesions. A certified nuclear medicine specialist and a paediatric radiologist interpreted the 
DMSA scans and CECT, respectively, and recorded the lesion site (right or left) and location (upper pole, midportion, or lower pole). If the patients underwent voiding cystourethrography (VCU) during the same hospitalization period, the results were also recorded.

Three certified radiologists (the first reader with 5 years of experience, the second reader with 10 years, and the third reader with 15 years) reviewed the B-mode, CDUS and MVUS images of the renal US examinations obtained in the same area of each kidney. The results of DMSA scans and CECT were given to three readers along with renal US images; however, the patients' information was blinded. The first and second readers had no experience with MVUS. They evaluated the following features independently without consensus: a) Lesion visibility on B-mode, CDUS, and MVUS: three radiologists decided whether an APN lesion was visible or not on each US image in three modes. They defined the US features of APN as follows: (1) a change in renal parenchymal echogenicity, (2) swelling of the renal parenchyma, (3) loss of renal corticomedullary differentiation, and (4) an area of hypoperfusion; b) Lesion distinguishability on the Doppler study: the radiologists compared CDUS and MVUS to evaluate which image better defined the extent and margin of the APN lesions; c) Diagnostic confidence in the Doppler study: the radiologists compared CDUS and MVUS to evaluate which image provided more diagnostic confidence for APN.

\section{Statistical analyses}

Lesion visibility: the results of three readers' interpretations were summated and the average lesion visibility was calculated. Then, the Cochran's Q test was used to analyse the statistical significance between the results of the three US modes.

Lesion distinguishability and diagnostic confidence in the Doppler study: the results of the three readers' interpretations were summated. Then, an $\mathrm{R}$ program coded-proportion test was used to analyse the statistical significance of the results.

Interobserver agreement: As three radiologists evaluated the US images independently, the interobserver agreement between them was calculated by Fleiss Kap$\mathrm{pa}$, and the strength was interpreted as follows: poor agreement for kappa values below zero, slight for kappa values of $0.01-0.20$, fair for kappa values of $0.21-0.40$, moderate for kappa values of $0.41-0.60$, substantial for kappa values of $0.61-0.81$, and almost perfect agreement for kappa values of $0.81-1.00$.

The statistical analyses were performed with the $\mathrm{R}$ program (version 3.6.1; package RVAideMemoire for Cochran Q test; package irr for Fleiss Kappa). Statistical significance was defined as a $p$ value of $<0.05$.

\section{Results}

All patients were admitted for fever. Among 41 APN lesions, all lesions were verified by DMSA scans and three lesions were verified by CECT as well. US examinations were performed on the admission day for five lesions, the day after admission for 28 lesions, two days after admission for six lesions and four and five days later in the remaining two lesions. Most of the DMSA scans and CECTs were performed after US, except in one patient where the DSMA scan and CECT were performed on the day of admission and the US examination was done five days later. The average interval was 1.4 days between US and DMSA in 41 lesions, and -1.7 days between US and CECT in 3 lesions.

The site and location of the 41 APN lesions were as follows: the upper pole was most affected with 30 lesions (73.2\%, 14 in the right kidney, 16 in the left kidney), followed by 9 lesions in the lower pole $(21.9 \%, 2$ in the right, 7 in the left) and 2 lesions in the mid-portion (4.9\%, 1 in each). The left kidney was more affected than the right kidney ( $58.5 \%$ vs. $41.5 \%)$.

VCU was performed in $37(90.2 \%)$ out of 41 APN lesions, which showed ipsilateral vesicoureteral reflux in 10 lesions.

\section{Lesion visibility}

Among 41 APN lesions, $52.8 \%$ were visible on Bmode, $85.4 \%$ on CDUS and $94.3 \%$ on MVUS. Cochran's Q test analysed the statistical significance between the results in the three US modes and the lesion visibility was superior in MVUS $(\mathrm{p}<0.001)$ (Table I, fig 2, fig 3).

\section{Lesion distinguishability}

MVUS was better at defining the extent and margins of the APN lesions than CDUS in $41.5 \%$ of the APN lesions. CDUS was better than MVUS in $6.5 \%$ of the lesions. The two modes showed equal distinguishability in

Table I. Result of Cochran's Q test for lesion visibility

\begin{tabular}{llll}
\hline Comparison & $\begin{array}{l}\text { B-mode } \\
\text { vs. CDUS }\end{array}$ & $\begin{array}{l}\text { B-mode } \\
\text { vs. MVUS }\end{array}$ & $\begin{array}{l}\text { CDUS } \\
\text { vs. MVUS }\end{array}$ \\
\hline$\chi^{2}$ & 34.8 & 47.3 & 3.37 \\
df & 1 & 1 & 1 \\
adjusted $p$ value & $<0.001$ & $<0.001$ & $<0.001$ \\
\hline
\end{tabular}

CDUS: colour Doppler US; MVUS: microvascular Doppler US

Table II. Result of proportion test for lesion distinguishability

\begin{tabular}{lll}
\hline Comparison & CDUS $>$ MVUS & CDUS $=$ MVUS \\
\hline MVUS $>$ CDUS & $<0.001$ & 0.35 \\
MVUS $=$ CDUS & $<0.001$ & - \\
\hline
\end{tabular}

CDUS: colour Doppler US, MVUS: microvascular Doppler US. Inequality signs mean superiority of lesion distinguishability. 


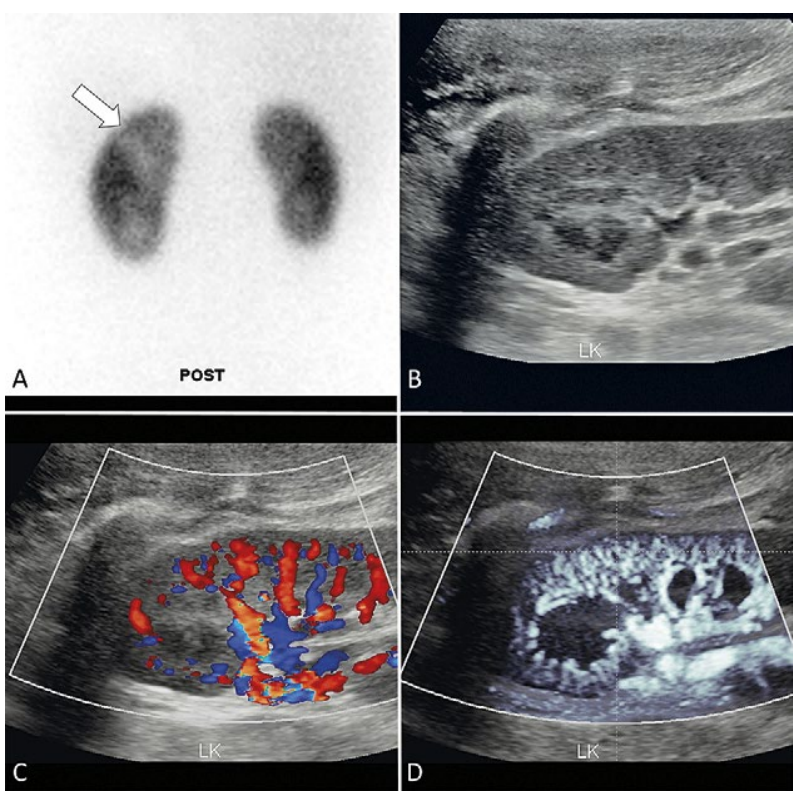

Fig 2. A 12-year-old girl with febrile UTI. (A) DMSA scan confirmed APN in the upper (arrow) and lower poles of the left kidney. B-mode (B), colour Doppler (C), and microvascular Doppler (D) for the upper pole lesion. All three readers interpreted an APN lesion seen on C and D, but not B. All of them reported that the lesion distinguishability and diagnostic confidence between $\mathrm{C}$ and $\mathrm{D}$ were equal.

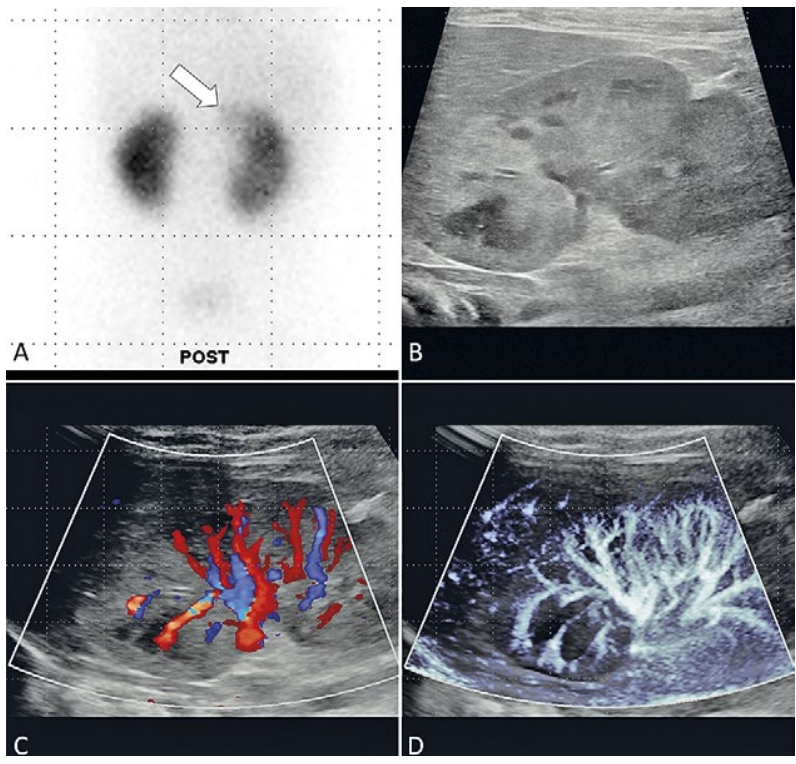

Fig 3. A 5-month-old girl with febrile UTI. (A) DMSA scan confirmed APN in the upper pole of the right kidney (arrow). B-mode (B), colour Doppler (C), and microvascular Doppler (D) for the upper pole lesion. All three readers interpreted an APN lesion seen on the B, C, and D. One of them reported that $\mathrm{D}$ showed better lesion distinguishability and more diagnostic confidence than $\mathrm{C}$, but two readers reported that the lesion distinguishability and diagnostic confidence were equal in both $\mathrm{C}$ and D.
$52 \%$ of the lesions. The $\mathrm{R}$ program coded-proportion test concluded that MVUS was superior to CDUS in distinguishing APN lesions ( $\mathrm{p}<0.001)$ (Table II, fig 4).

\section{Diagnostic confidence}

The diagnostic confidence was higher for MVUS than CDUS in $36.6 \%$ of APN lesions, higher for CDUS than MVUS in $4.9 \%$, and equal in the remaining $58.5 \%$. Although the diagnostic confidence between CDUS and MVUS was equal in $56.1 \%$ of the 41 APN lesions, MVUS was preferred in $36.6 \%$, whereas CDUS was preferred in only $4.9 \%$ of the APN lesions, meaning that the three readers preferred MVUS over 7 times more when the two modes of Doppler images were provided simultaneously. The $\mathrm{R}$ program coded-proportion test concluded that MVUS provided more confidence than CDUS for the diagnosis of paediatric APN $(p<0.001$ for MVUS $>$ CDUS, $p=0.02$ for MVUS $=$ CDUS) (Table III, fig 5).

\section{Interobserver agreements}

The results of the Fleiss Kappa analysis of the three readers' agreement are as follows: moderate agreement for lesion visibility in B-mode (0.576) and CDUS (0.414), slight agreement in MVUS (0.091), fair agreement for lesion distinguishability (0.324), and slight agreement for confidence of diagnosis (0.118).

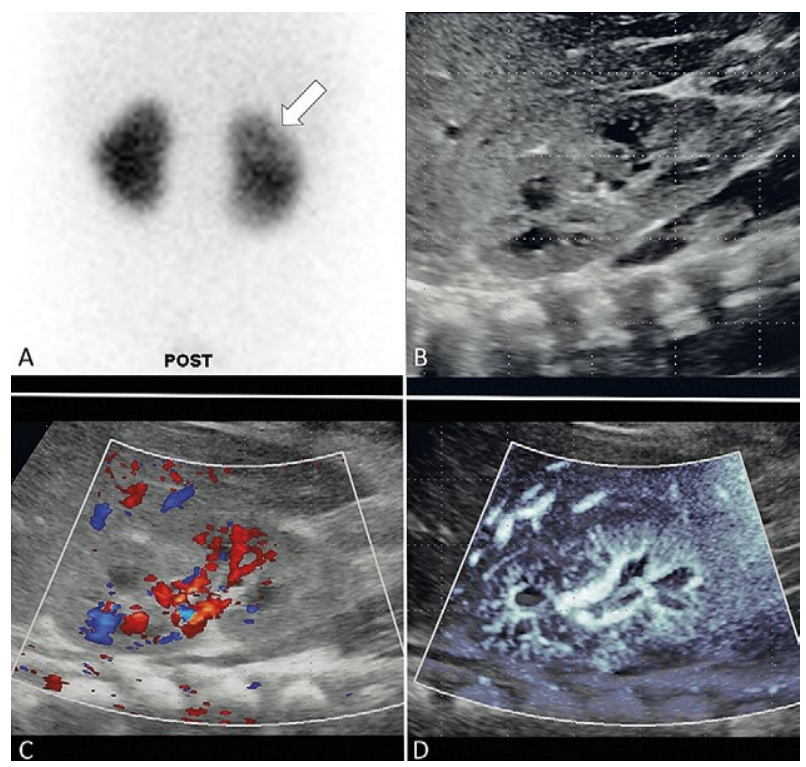

Fig 4. A 5-month-old boy with febrile UTI. (A) DMSA scan confirmed APN in the upper pole of the right kidney (arrow). Bmode (B), colour Doppler (C), and microvascular Doppler (D) for the upper pole lesion. Two readers interpreted an APN lesion seen on $\mathrm{C}$ and $\mathrm{D}$, and one reader interpreted an APN lesion seen on B, C, and D. All of them reported better lesion distinguishability on $\mathrm{D}$ than $\mathrm{C}$. Two of them reported that $\mathrm{D}$ provided more diagnostic confidence than $\mathrm{C}$, and one of them reported that the diagnostic confidence as equal between $\mathrm{C}$ and $\mathrm{D}$. 
Table III. Result of proportion test for diagnostic confidence

\begin{tabular}{lll}
\hline Comparison & CDUS $>$ MVUS & CDUS $=$ MVUS \\
\hline MVUS $>$ CDUS & $<0.001$ & 0.02 \\
MVUS $=$ CDUS & $<0.001$ & - \\
\hline
\end{tabular}

CDUS: colour Doppler US, MVUS: microvascular Doppler US. Inequality signs mean superiority of diagnostic confidence.

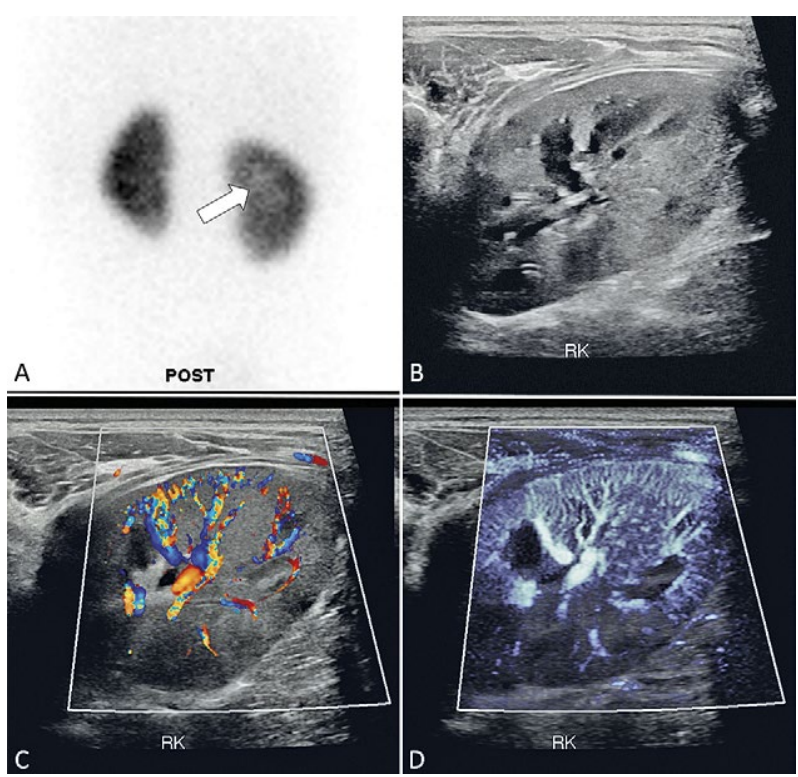

Fig 5. A 17-month-old boy with febrile UTI. (A) DMSA scan confirmed APN in the mid-portion of the right kidney (arrow). B-mode (B), colour Doppler (C), and microvascular Doppler (D) for the mid-portion lesion. All three readers interpreted an APN lesion seen on B, C, and D. Two of them reported that $\mathrm{D}$ showed better lesion distinguishability than $\mathrm{C}$, and one of them reported as equal. Two of them reported that $\mathrm{D}$ provided more diagnostic confidence than $\mathrm{C}$, and one of them reported as equal.

\section{Discussion}

The US findings of APN are changes in renal parenchymal echogenicity, swelling of the renal parenchyma, loss of renal corticomedullary differentiation, urothelial thickening of the renal pelvis and ureter and an area of decreased perfusion, which may involve the kidneys focally or diffusely $[8,12]$. As US can also provide a structural overview of the renal anatomy and rule out hydronephrosis, abscess or calculus, it is worthwhile as a primary imaging modality for APN in children [13,14]. However, these US findings were not always well-characterized, and a number of papers have reported better US techniques for diagnosing paediatric APN including B-mode, colour Doppler, power Doppler and contrastenhanced US [2,15-18]. Power Doppler US (PDUS) has been tried for the past 14 years as a method for assessing more renal vascular structures and achieving better sen- sitivity for detecting renal blood flow than CDUS. Therefore, PDUS showed superior detectability of APN represented as hypoperfused areas in the kidneys. Recently, a third-generation Doppler US technique, MVUS, has emerged to depict spatially small and temporally slow blood flow using low pulse repetition frequency with an advanced clutter filter that can differentiate microvascular flow from clutter artifacts [10,19]. Despite the relative novelty of microvascular imaging, Some studies already have reported its strength and clinical benefits in various organs including liver, breast, thyroid gland, ovary, testis, brain, kidney, GI tract, lymph node, extremities and carotid plaques $[10,11,20,21]$.

In kidneys, MVUS has shown the superb ability to visualize more detailed branching patterns of interlobar, arcuate and interlobular arteries to the renal cortex, compared to CDUS or PDUS [10,22,23]. Additionally, contrast-enhanced US has emerged as a promising alternative to DMSA with high sensitivity and specificity for diagnosing APN, yet it still requires intravenous administration of US contrast media and not enough studies reported for clinical application in children [24].

In this study, we evaluated APN lesion visibility using MVUS technique called MV-Flow ${ }^{\mathrm{TM}}$ and compared it to B-mode and CDUS. We found that MVUS showed better visibility of APN lesions than B-mode and CDUS and provided better depiction and better distinguishability of minute hypoperfused areas compared to CDUS. Since the main pathophysiology of APN is hypoperfusion at the level of microvessels including cortical arteriolar vasoconstriction and peritubular capillary occlusion by inflammatory cells, imaging of perfusion by minute vessels using advanced techniques such as MVUS are helpful in the diagnosis of APN. Thus, this study revealed that the feasibility of MVUS to detect APN lesions was superior to that of CDUS. Further studies with MVUS could extend its clinical value for many vascular indications, including renal infarction, renal cortical necrosis, renal tumours, chronic renal disease, acute tubular necrosis, renal transplantation, as well as acute pyelonephritis.

US examination is recognized as an operator-dependent technique. The artifacts or factors degrading the quality of paediatric US, such as motion, crying and bowel gas could be improved according to the radiologists' experience and know-how. To minimize operatordependent issues in this study, the most experienced radiologist performed all US scans including the B-mode, CDUS and MVUS scans. Despite our expectations, the Fleiss Kappa values were all less than 0.6, which meant the interobserver agreement was not perfect. For example, the lesion visibility in MVUS showed the highest result (94.3\%). However, the kappa value among the three 
readers was the lowest $(0.091)$. We suggest that these conflicting results were due to the lack of experience with MVUS in two readers and the independent interpretation of the three readers without consensus. The highest kappa value was seen when the readers interpreted the Bmode images, which were the basic and most familiar US images to all three radiologists. We could expect that the agreement would have been higher if more of the readers were familiar with MVUS images.

There were several limitations to our study. First, the data were retrospectively obtained. Therefore, we could only enrol a small number of patients. Second, the second-generation Doppler US technique, PDUS, was not obtained in this study. The advantage of MVUS would be reinforced if a comparison between PDUS and MVUS was presented. Third, we did not enrol the patients affected by UTI without APN lesion. Fourth, all US examinations in our study were performed by one paediatric radiologist. We expect that further studies performed by more radiologists aware of MVUS may show better agreement between the observers.

In conclusion, MVUS showed improved detectability of hypoperfused areas in paediatric APN compared to CDUS and increased US diagnostic performance of APN without radiation exposure. The more sensitive diagnosis of childhood APN is feasible with the new Doppler US technique, MVUS. Confident, early diagnosis of APN using MVUS may help reduce additional radiation exposure and also enable proper treatment to prevent chronic sequelae of the kidneys.

Acknowledgments: This study was supported by the Korea University (K1810931).

\section{Conflict of interest: none}

\section{References}

1. Majd M, Nussbaum Blask AR, Markle BM, et al. Acute pyelonephritis: comparison of diagnosis with 99mTc-DMSA, SPECT, spiral CT, MR imaging, and power Doppler US in an experimental pig model. Radiology 2001;218:101-108.

2. Stogianni A, Nikolopoulos P, Oikonomou I, et al. Childhood acute pyelonephritis: comparison of power Doppler sonography and Tc-DMSA scintigraphy. Pediatr Radiol 2007;37:685-690.

3. Vourganti S, Agarwal PK, Bodner DR, Dogra VS. Ultrasonographic evaluation of renal infections. Radiol Clin North Am 2006;44:763-775.

4. Das CJ, Ahmad Z, Sharma S, Gupta AK. Multimodality imaging of renal inflammatory lesions. World J Radiol 2014;6:865-873

5. Ha SK, Seo JK, Kim SJ, et al. Acute pyelonephritis focusing on perfusion defects on contrast enhanced computer- ized tomography(CT) scans and its clinical outcome. Korean J Intern Med 1997;12:122-127.

6. Hwang M, Piskunowicz M, Darge K. Advanced Ultrasound Techniques for Pediatric Imaging. Pediatrics 2019; 143:e20182609.

7. Lavocat MP, Granjon D, Allard D, Gay C, Freycon MT, Dubois F. Imaging of pyelonephritis. Pediatr Radiol 1997;27:159-165.

8. Craig WD, Wagner BJ, Travis MD. Pyelonephritis: radiologic-pathologic review. Radiographics 2008;28:255-277.

9. Park AY, Seo BK. Up-to-date Doppler techniques for breast tumor vascularity: superb microvascular imaging and contrast-enhanced ultrasound. Ultrasonography 2018;37:98106.

10. Yoo J, Je BK, Choo JY. Ultrasonographic Demonstration of the Tissue Microvasculature in Children: Microvascular Ultrasonography Versus Conventional Color Doppler Ultrasonography. Korean J Radiol 2020;21:146-158.

11. 11. Jiang ZZ, Huang YH, Shen HL, Liu XT. Clinical applications of superb microvascular imaging in the liver, breast, thyroid, skeletal muscle, and carotid plaques. J Ultrasound Med 2019;38:2811-2820.

12. Chung EM, Soderlund KA, Fagen KE. Imaging of the Pediatric Urinary System. Radiol Clin North Am 2017;55:337357.

13. Subcommittee on Urinary Tract Infection. Steering Committee on Quality Improvement and Management. Roberts KB. Urinary tract infection: clinical practice guideline for the diagnosis and management of the initial UTI in febrile infants and children 2 to 24 months. Pediatrics 2011;128:595-610.

14. Bae HJ, Park YH, Cho JH, Jang KM. Comparison of 99mTc-DMSA Renal Scan and Power Doppler Ultrasonography for the Detection of Acute Pyelonephritis and Vesicoureteral Reflux. Child Kidney Dis 2018;22:47-51.

15. Dacher JN, Pfister C, Monroc M, Eurin D, LeDosseur P. Power Doppler sonographic pattern of acute pyelonephritis in children: comparison with CT. AJR Am J Roentgenol 1996;166:1451-1455.

16. Sakarya ME, Arslan H, Erkoc R, Bozkurt M, Atilla MK. The role of power Doppler ultrasonography in the diagnosis of acute pyelonephritis. Br J Urol 1998;81:360-363.

17. el Hajjar M, Launay S, Hossein-Foucher C, Foulard M, Robert Y. Power Doppler sonography and acute pyelonephritis in children: comparison with Tc-DMSA scintigraphy. Arch Pediatr 2002;9:21-25.

18. Bykov S, Chervinsky L, Smolkin V, Halevi R, Garty I. Power Doppler sonography versus Tc-99m DMSA scintigraphy for diagnosing acute pyelonephritis in children: are these two methods comparable? Clin Nucl Med 2003;28:198-203.

19. Jung HK, Park AY, Ko KH, Koh J. Comparison of the diagnostic performance of power Doppler ultrasound and a new microvascular Doppler ultrasound technique (AngioPlus) for differentiating benign and malignant breast masses. J Ultrasound Med 2018;37:2689-2698.

20. Ohno Y, Fujimoto T, Shibata Y. A new era in diagnostic ultrasound, superb microvascular imaging: preliminary 
results in pediatric hepato-gastrointestinal disorders. Eur J Pediatr Surg 2017;27:20-25.

21. Ayaz E, Aslan A, İnan İ, Y1kılmaz A. Evaluation of Ovarian Vascularity in Children by Using the "Superb Microvascular Imaging" Ultrasound Technique in Comparison With Conventional Doppler Ultrasound Techniques. J U1trasound Med 2019;38:2751-2760.

22. Quaia E, Correas JM, Mehta M, Murchison JT, Gennari AG, van Beek EJR. Gray scale ultrasound, color Doppler ultrasound, and contrast-enhanced ultrasound in renal parenchymal diseases. Ultrasound Q 2018;34:250-267.

23. Correas JM, Anglicheau D, Joly D, Gennisson JL, Tanter $\mathrm{M}$, Helenon O. Ultrasound-based imaging methods of the kidney-recent developments. Kiney Int 2016;90:11991210.

24. Jung HJ, Choi MH, Pai KS, Kim HG. Diagnostic performance of contrast-enhanced ultrasound for acute pyelonephritis in children. Sci Rep 2020;10:10715. 